\title{
Fiber laser design and measurements for fiber optic hydrophones in their application for ultra-high energy neutrino detection
}

\author{
V. M. Baas* \\ TNO Technical Sciences, P.O. Box 155, 2600 AD Delft, The Netherlands \\ The Hague University of Applied Sciences, P. O. Box 13336, 2501 EH, Den Haag, The \\ Netherlands \\ E-mail: vincent.baas@tno.nl \\ P. Toet ${ }^{\dagger}$ \\ TNO Technical Sciences, P.O. Box 155, 2600 AD Delft, The Netherlands \\ E-mail: peter.toetatno.nI

\section{E. J. Buis} \\ TNO Technical Sciences, P.O. Box 155, 2600 AD Delft, The Netherlands \\ E-mail: ernst-jan.buisetno.nl
}

\begin{abstract}
Due to the low expected flux, the detection of ultra-high energy neutrinos with energies above $10^{18} \mathrm{eV}$ requires a neutrino telescope that is at least an order of magnitude larger than what has been achieved today. A potential technology for a large scale neutrino telescope, which is sensitive enough to detect the low thermoacoustic signals induced by the cosmic rays in water, is offered by fiber optical hydrophones. Optical fibers form a natural way to create a distributed sensing system in which several pressure sensitive transducers are attached to a single fiber. At the position of each transducer, a fiber laser is introduced which forms a local coherent light source. In this way a low cost, low power sensor network can be deployed in the abyss that can be scaled to the required volume of a neutrino telescope. The design of one of the key components, the fiber laser, is discussed. Both DFB and DBR laser types were considered and their compatibilty with the transducers have been investigated, as well as their performance when operated in a serial multiplexing mode.
\end{abstract}

The 34th International Cosmic Ray Conference,

30 July- 6 August, 2015

The Hague, The Netherlands

\footnotetext{
* Speaker.

† Corresponding author
} 


\section{Introduction}

Ultra-high energy (UHE) neutrinos, at an energy of $>10^{18} \mathrm{eV}$ may crucial information on the underlying principles of the GZK [1] cut-off mechanism, but also on distant astrophysical sources [2]. Since only very few UHE neutrinos reach earth, their detection require a telescope with a detector volume of circa $100 \mathrm{~km}^{3}$. When an ultra high energy (UHE) neutrino interacts with a proton in water a cascade of secondary particles will be produced. This cascade will have the shape of a cone that has a length in the order of $10 \mathrm{~m}$ and a diameter of $1 \mathrm{~cm}$ effectively forming a line source. The produced secondary particles will deposit their energy in the surrounding medium heating the water $[4,5]$. The sudden heating of the water will cause a pressure wave results in a bipolar signal with a frequency between a few and $50 \mathrm{kHz}$. A cascade from a $10^{18} \mathrm{eV}$ neutrino will cause an sound pressure level of $10 \mathrm{mPa}$ or $5 \mathrm{mPa}$ at a distance of $200 \mathrm{~m}$ and $500 \mathrm{~m}$, respectively [3]. To fully reconstruct the direction and energy of a cosmic ray event an array of hydrophones will be used. The hydrophones will therefore be placed in several vertical stories or strings of each about a $1 \mathrm{~km}$ long. It has been argued that fiber optic hydrophones would be a suited technology for realizing these strings as it provides sensitivity with the feasibility to create a large sensor network [6].

The aim of this paper is to discuss one of key components of fiber optic hydrophones, which is the fiber laser. We will investigate different designs for the fiber laser and their design parameters, such as gain and output laser output power both individually as well as in a multiplexed array.

\section{Fiber lasers for optical hydrophones}

A fiber optical hydrophone consists of a transducer to convert the pressure signal to a strain signal and a local coherent light source, which is formed by a fiber laser. Sound that falls on the transducer will be converted to strain in the fiber laser which results in a wavelength shift of the laser. The shift in wavelength is then a measure for the sound pressure. Because the sound pressure level is quite low the corresponding wavelength shift will also be small. To measure this small wavelength shift an interferometric interrogation unit will have to be used. The current transducer as developed by TNO is designed to measure acoustic signals up to $40-50 \mathrm{kHz}$. This sensor consists of two curved metal membranes which are placed $6 \mathrm{~mm}$ apart from each other [7]. A fiber containing the fiber laser will be located in between these two membranes. The size of the sensor limits the size of the attached fiber laser, but presently no commercially available fiber laser exists that has a length of less than $6 \mathrm{~mm}$. To our knowledge, the shortest DBR laser is $7 \mathrm{~mm}$ [9] and the shortest $\pi$-shifted DFB laser $17 \mathrm{~mm}$ [10]. Both these fiber lasers were manufactured and tested in a laboratory setup by A.C.L. Wong et al. (2011). Therefore a new fiber laser design is required with a stable lasing operation and that is compatible with the available transducer.

\section{DBR and DFB fiber lasers}

A linear fiber laser consists of two reflectors and a gain medium, a rare earth element doped fiber. The reflectors are formed by fiber Bragg reflectors (FBGs) and the gain medium consists of an erbium doped fiber. As shown in figure 1 two types fiber laser exists that differ by the distance 
between the two reflectors and hence the effective cavity length. When the cavity length is short and exactly half the period of the grating the laser is considered $\pi$-shifted and the design is called a $\pi$-shifted Distributed Feedback (DFB) laser. This variant is schematically depicted in figure 1 (a). If the length of the cavity is in the order of the length of the reflectors the fiber laser is called a Distributed Bragg Reflector (DBR) laser which can be seen in figure 1 (b). Commonly, the $\pi$-shifted DFB laser is preferred as it has the lowest possible laser gain threshold and it is less vulnerable to mode hopping.

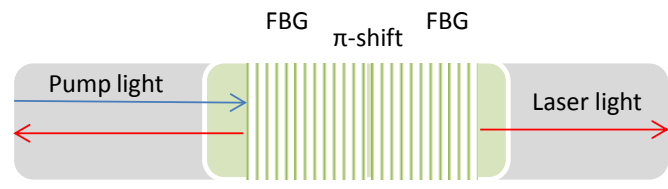

Doped fiber

(a)

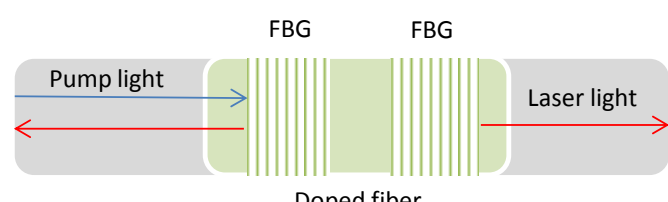

(b)

Figure 1: Principle of a $\pi$-shifted DFB laser (a) and a DBR laser (b).

Note that in figure 1 the laser is pumped optically from the left while laser light is emitted to both sides. The configuration in which the laser light is collected at the same side from as where the pump laser light is injected in the fiber laser is called backwards pumping. If the fiber laser light is collected from the opposite side, the configuration is called forward pumping. If the laser is pumped from both sides, the configuration is called double pumping. This definition of pumping directions is used when the multiplexing of several lasers is considered (section 5). Note that when the fiber lasers are multiplexed the output power at the interferometer has to be at least $10 \mu \mathrm{W}$ per fiber laser for good performance of the interrogator in terms of signal to noise ratio.

\section{Fiber laser design}

The erbium that forms the dopant in the gain medium of the fiber laser can be brought in an excited state (pumping) and will subsequently emit light between 1530 and $1570 \mathrm{~nm}$. The emitted laser light is then depending on the exact wavelength that the FBGs will reflect. The erbium emission and absorption curves can be seen in figure 2. The main reason that erbium is chosen is the absorption in single mode silica based fibers which is the lowest possible value at $1550 \mathrm{~nm}$ so light around these wavelengths can be transported over long distances without being attenuated. An erbium gain medium will be pumped at $976 \mathrm{~nm}$ or $1480 \mathrm{~nm}$. Pumping at $1480 \mathrm{~nm}$ is preferred when the pump power has to be transported over long distances. The absorption in a standard single mode optical fiber (type Corning SMF28) is circa $1 \mathrm{~dB} / \mathrm{km}$ at $976 \mathrm{~nm}$ and only circa $0.23 \mathrm{~dB} / \mathrm{km}$ at $1480 \mathrm{~nm}$.

For the purpose of laser design a simulations package was designed that allowed to simulate the reflection of FBG as well as other relevant parameters of fiber lasers, such as gain threshold and pump and laser output power. The simulations were verified using measurements on a nonapodized $\pi$-shifted DFB laser that was available at TNO and used in a previous version of the fiber hydrophone [6]. This laser has a cavity length of $20 \mathrm{~mm}$. In figure 3 (a) the results of a reflection spectrum simulation (dotted line) is compared to the measured spectrum (solid line). The simulated 


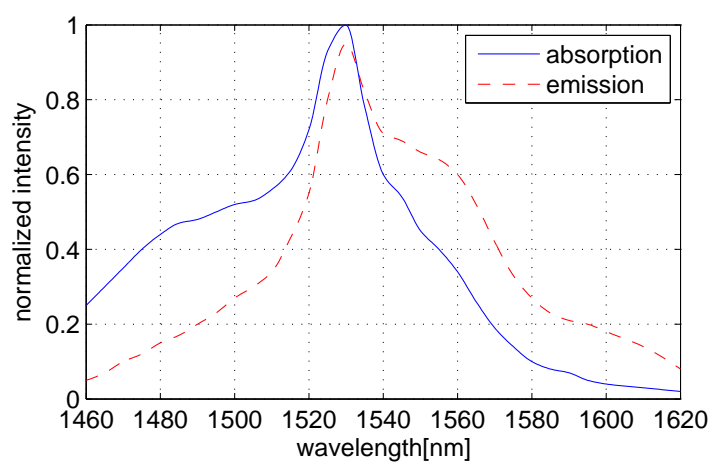

Figure 2: Emission and absorption of erbium as a function the wavelength.

side lobes are slightly higher than the measured ones, this is most likely the result of grating errors. The laser was made in a low doped fiber and has an equal length distribution. In figure 3 (b) the output power of the laser is plotted as a function of the input power to get the slope efficiency of the laser. A straight line fit to the data provides information on the dopant concentration $(4.8 \mathrm{~dB} / \mathrm{m})$ of the fiber laser, which is close to the expected value.

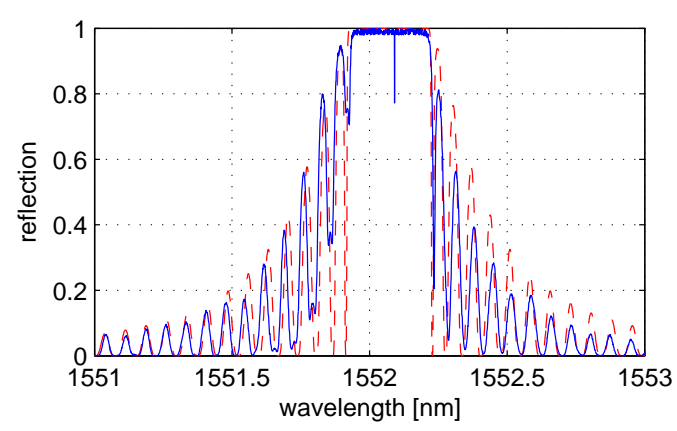

(a)

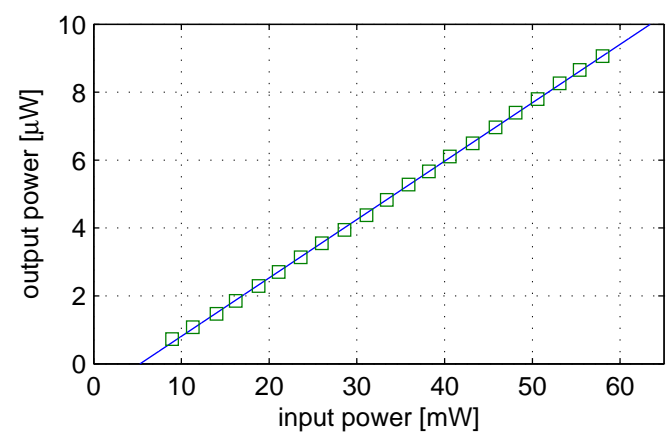

(b)

Figure 3: (a) Measured and simulated reflection spectrum of a $20 \mathrm{~mm}$ DFB fiber laser. This laser has a wavelength of about $1552.1 \mathrm{~nm}$. In (b) the measured slope efficiency of the fiber laser is plotted.

Since the $20 \mathrm{~mm}$ cavity length is too long for its application in the update version of the hydrophone [7], alternative (and shorter) designs were investigated. Therefore a design was made of a $5 \mathrm{~mm}$ long $\pi$-shifted DFB laser of which the reflection spectrum is plotted in figure 4 . The design of the laser has a length of $5 \mathrm{~mm}$, a peak absorption of $40 \mathrm{~dB} / \mathrm{m}$ at $1530 \mathrm{~nm}$ (a high doping concentration), an index modulation of $6.8 \cdot 10^{-4}$ and both FBGs had equal length. Using the reflection spectrum and the dopant concentration, the stability laser can be determined. The fiber laser is considered stable when the the supplied gain is above the required laser gain. The laser gain threshold is determined by the nominal cavity length $\left(l_{c}\right)$ and the reflection of the two FBGs. 
The gain threshold $\left(g_{\text {th }}\right)$ of the fiber laser is given by:

$$
g_{\text {th }}=\frac{1}{2 l_{c}} \ln \left(\frac{1}{R_{L} R_{R}}\right)+a_{o}
$$

where $R_{L}$ and $R_{R}$ are the reflectivity of the FBG on the left and right side of the cavity and $a_{o}$ is the background loss of the laser light. The geometry of the fiber laser and in particular the cavity length determines the laser wavelength range in which a stable lasing operation is established and was determined as follows. A typical gain of a erbium doped fiber is $g_{\mathrm{m}}=40 \mathrm{~dB} / \mathrm{m}$. The emission curve in figure 2 determines the gain of the laser at each wavelength. In figure 4 (b) the gain of the medium is plotted as a function of the wavelength together with the gain threshold of the fiber laser. To determine the gain threshold a design was made of the grating structure at several wavelength. The reflectivity coefficients $R_{L}$ and $R_{R}$ were determined to get the gain threshold as defined in equation 4.1. From the figures 2 and 4 (b) we can infer the useful range of the erbium

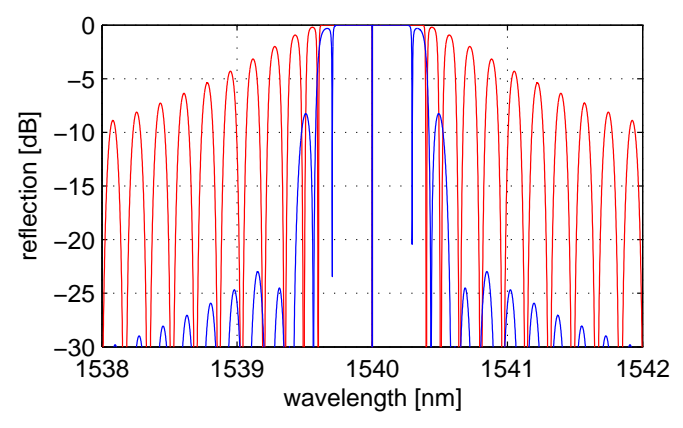

(a)

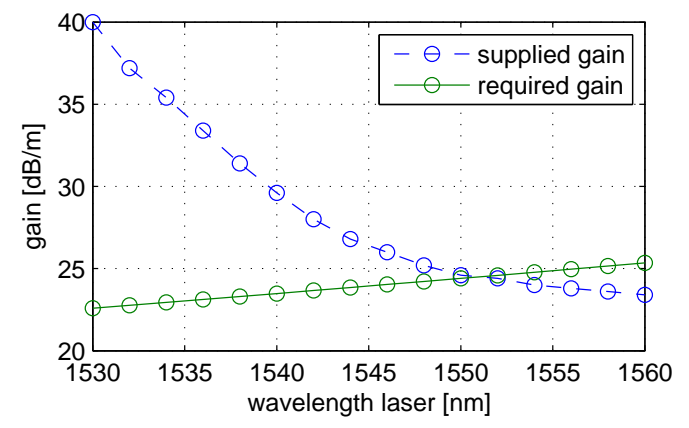

(b)

Figure 4: (a) An example of a simulated reflection spectrum of the a $5 \mathrm{~mm}$ DFB laser. The reflectivity is shown for an apodized (blue) and a non-apodized grating (red). The wavelength of the fiber laser is about $1540 \mathrm{~nm}$. In (b) the supplied gain and gain threshold as a function of wavelength is plotted.

emission spectrum. The lower range of the spectrum is determined to be $\lambda_{\text {low }}=1530 \mathrm{~nm}$, because the emission is larger than the absorption. The upper end of the range is determined by the gain threshold, which should be lower than the supplied gain, which is determined to be $\lambda_{\text {high }}=1550 \mathrm{~nm}$.

To multiplex this fiber laser (section 5), apodization will have to be applied to decrease the side lobes. In figure 4 (double Gaussian) apodization (solid line) is compared to constant index modulation (dotted line) for this design. This will decrease the side lobes to $-30 \mathrm{~dB}, 2 \mathrm{~nm}$ from the laser wavelength allowing a maximum distance of $100 \mathrm{~m}$ between the fiber lasers. The maximum distance between the hydrophones is limited by the optical feedback [8]. Optical feedback is coupling between the laser cavity and the external cavity, which is formed by neighboring lasers on a fiber. The effect gives rise to unwanted phenomena, such as intensity and frequency noise, multiple frequency oscillations and self-pulsation [11]. The feedback parameter $C$, which denotes the strength of optical feedback is given [8]:

$$
C \approx 4 \sqrt{1+\alpha^{2}} \kappa L_{\mathrm{ext}} \exp (-\kappa L) r_{\mathrm{ext}}
$$

where $\alpha$ is the Henry factor (line width enhancement), $L$ is the length of the laser cavity, $\kappa$ is the grating strength and $r_{\mathrm{ext}}$ is the external reflectivity coefficient. $\alpha$ is taken as unit, while the 
other parameters follow from the grating design. As a rule of thumb, to avoid optical feedback the coefficient $C$ should be kept to $C<1$. For the grating under design this means that the external cavity $L_{\text {ext }}$ (or distance between neighboring hydrophones) should be less than $100 \mathrm{~m}$. The total length will have to be increased so the strain sensitivity will decrease to $0.6 \mathrm{pm} / \mu \varepsilon$.

\section{Multiplexing fiber lasers in a large sensor network}

Next, we investigated the use of the fiber laser as described in the previous section in a serially multiplexed arrangement. As motivated above we kept the interspacing of the fiber lasers to 100 meters. Although multiplexing of fiber lasers has been achieved before, see e.g. [12], we investigate here the limits that are introduced by a really short fiber laser design. In figure 5 we draw the schematics of a number of hydrophones that are serially multiplexed on a single fiber. The figure shows the sources for the light losses, which are expected to have an impact on the pumping design of the fiber. Also shown in figure ?? is that each laser will have a different wavelength, so by

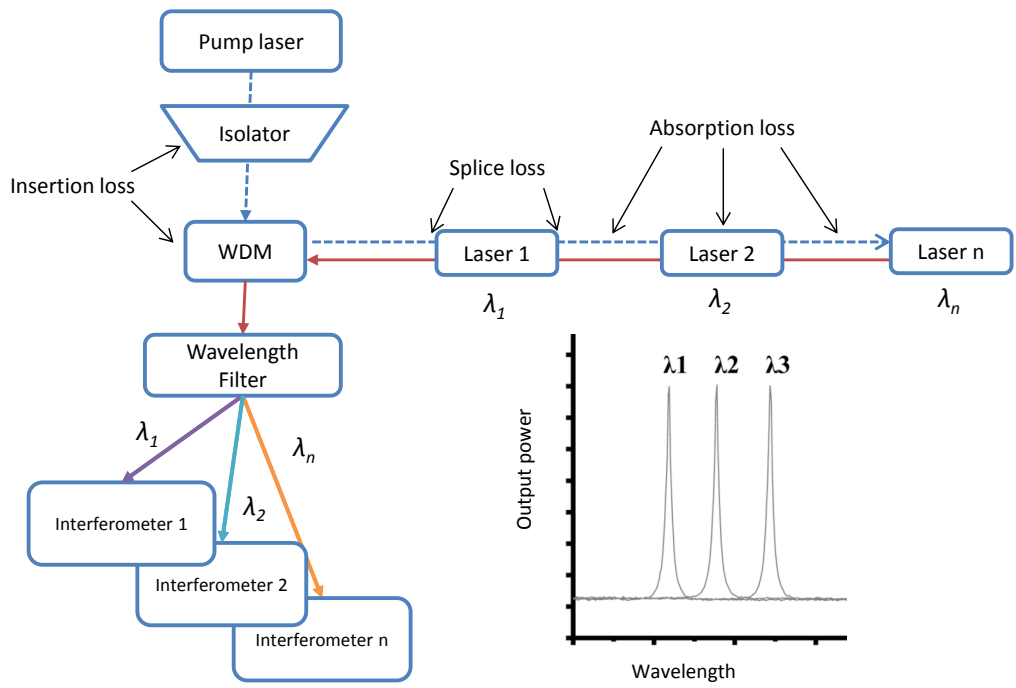

Figure 5: Schematics of a multiplexed array of hydrophones. Indicated in the figure as well are the light losses.

filtering out the different wavelengths at the interrogation unit all the hydrophones can be read out.

The pump power at each laser in array of 10 fiber lasers can be seen in figure 6 (a), where the output power of the pump laser is $100 \mathrm{~mW}$. When the array is pumped backwards the pump power decreases throughout the array and every next fiber laser in the array will receive less power. The pump power will decrease due to insertion losses of the isolator, WDM coupler and absorption and splice losses in both the connecting and the erbium doped fibers. This problem is the same with forwards pumping. In double pumping the pump power will be almost constant throughout the array.

In figure 6 (b) the expected laser power at the interrogation unit is given for the different pumping strategies. We assumed here a $1 \mathrm{~km}$ lead fiber between the wavelength division multiplexer (WDM coupler) and the first laser. The distance between the fiber lasers is $100 \mathrm{~m}$ (using 


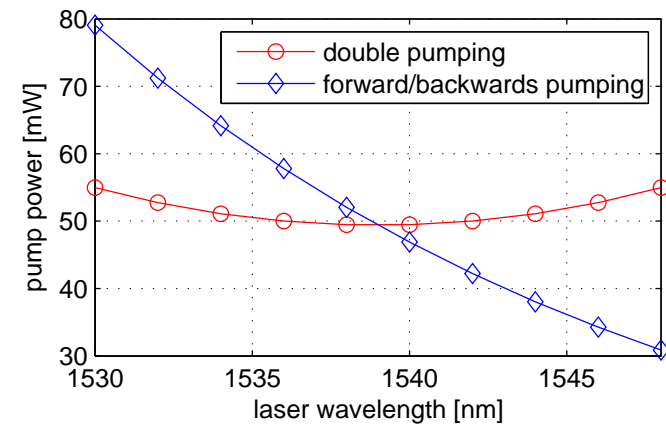

(a)

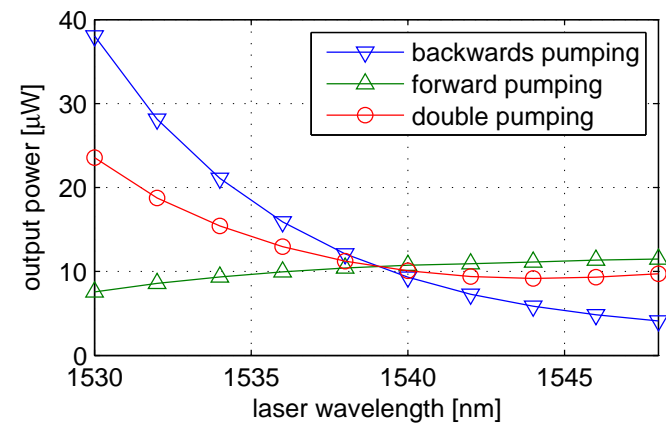

(b)

Figure 6: (a) Pump power achieved in the array as function of the hydrophone position (laser wavelength). In (b) the fiber laser power at the interrogator is plotted for the multiplexed hydrophones for three different pump strategies.

SMF28). As can be seen from the figure, the situation in which the array is pumped forward, the laser light is almost equal for all fiber lasers in the string. This is opposite to what is expected from figure 6 (a), where it is shown that all fiber lasers received (almost) equal pump power. Note that despite a relatively equal distribution of the pump power over the fiber lasers in case of double pumping, the returned fiber laser power is not. This is due to both the variance of the pump threshold and the slope efficiency with laser wavelength, which vary as a function of wavelength and hence from fiber laser to fiber laser.

\section{Conclusions}

Fiber lasers are an important component of a fiber optic hydrophone, which may provide the hydrophone technology the proper sensitivity for its application in an acoustic neutrino telescope. We have carried out a design study of the fiber lasers and investigated in the feasibility of multiplexing several fiber lasers on a single optical fiber. An important boundary condition for this study was the maximum length of the fiber laser structure, i. e. $5 \mathrm{~mm}$, which is derived from the design of the mechanical transducer. The dimensions of the transducer is given by the expected frequency range in which the acoustic signal from cosmic neutrinos is expected.

Based on the study as described in this paper we arrive at the following conclusions. The optimum spectral range in which the lasers operate is between 1530 and $1550 \mathrm{~nm}$. Although the emission spectrum of erbium is much broader, the used range is limited by, on the low side by the self-absorption of erbium and on the upper end by the limited length of the grating structure.

We estimate that given the spectral range $(1530-1550 \mathrm{~nm})$, about 10 fiber lasers can be multiplexed on a single fiber. This number is limited by the apodization of the grating structure in the fiber laser. Forward pumping will be probably the best pumping strategy where all fiber laser have about the same output power. To comply with the current detection limit at the interrogation unit of $10 \mu \mathrm{W}$ either the ratio between the two sides has to be made unequal (to achieve a higher slope efficiency) or the the initial pump power will have to be increased. 
It should be noted that the design driver is this study is the dimension of the transducer as it limits the length of the grating structure in the fiber. This has far reaching consequences: it determines the effective wavelength range of the erbium emission that can be used, as well as the number of hydrophones that can be multiplexed on a single fiber.

\section{References}

[1] K. Greisen, Phys. Rev. Lett., End to the Cosmic-Ray Spectrum? Phys. Rev. Lett., 16 (1966) 748 ; G. T. Zatsepin and V. A. Kuzmin, Upper Limit of the Spectrum of Cosmic Rays, JETP Lett., 4 (1966) 78.

[2] E. Waxman. Neutrino astrophysics: A new tool for exploring the universe, Science, 315 (5808):6365, 2007.

[3] K. Graf, Acoustic neutrino detection in sea water: Technical aspects, AIP Conference Proceedings 1535 (2013) p169

[4] G. A. Askaryan, Acoustic recording of neutrinos, Zemlia i Vselennaia, 1 (1979) 13

[5] J. G. Learned, Acoustic radiation by charged atomic particles in liquids: An analysis Phys. Rev. D 19 (1979) 3293

[6] E. J. Buis et al, Fibre laser hydrophones for cosmic ray particle detection, Journal of Instrumentation, 9-03 (2014) C03051;

[7] E. J. Buis et al, Design studies for a neutrino telescope based on optical fiber hydrophones. these proceedings.

[8] G.A.Cranch et al., Distributed Feedback Fiber Laser Strain Sensors, IEEE SENSORS JOURNAL, vol. 8 , no. 7, (2008).

[9] A. C. L. Wong et al, Extremely short distributed Bragg reflector fiber lasers with subkilohertz linewidth and ultralow polarization beat frequency for sensing applications, Meas. Sci. Technol., vol. 22 (2011).

[10] A. C. L. Wong et al, Ultrashort Distributed Feedback Fiber Laser with SubKilohertz Linewith for Sensing Applications, Laser Physics, vol. 21, no. 1, (2011) p. 163.

[11] K. Peterman, External optical feedback phenomena in semiconductor lasers, IEEE J. Sel. Topics Quantum Electron, vol 1, no 2 (1995) p 480.

[12] Y. Leguillon et al, First demonstration of a 12 DFB fiber laser array on a $100 \mathrm{GHz}$ ITU grid, for underwater acoustic sensing application, Proc. SPIE, 8439 (2012) pp.84390J, 